\title{
Student Outcomes from Undergraduate Research Programs: Comparing Models of Research Experiences for Undergraduates (REUs)
}

D. Jake Follmer, Sarah Zappe, Esther Gomez, Manish Kumar,

Pennsylvania State University

\begin{abstract}
Programs involving research experiences for undergraduates (REUs) typically reflect either university-sponsored programs or programs funded by the National Science Foundation (NSF) that aim to facilitate students' researchbased skill development. Despite the prevalence of research supporting the effectiveness of these programs, little research has compared the programs or evaluated the impact of differing REU models on gains in student research skills. This article examines gains made in research-based skills and experiences by students who participated in a university-sponsored or an NSF-funded REU program in engineering at a large research university. Students completed measures of research-based experiences, openness to research collaboration, and likelihood of pursuing graduate school activities prior to and after completing the research programs. Students also rated the effectiveness of core REU elements at program completion. Students participating in both REU programs demonstrated significant gains in a measure of research-based experience. Students participating in the NSF-funded REU reported higher gains in specific research-based skills compared with students participating in the universitysponsored REU. Student ratings of the openness to research collaboration and likelihood of pursuing graduate school were comparable across REU programs.
\end{abstract}

Keywords: undergraduate research, STEM, underrepresented groups, REU programs, assessment, program evaluation

\section{doi: $10.18833 /$ spur/1/1/5}

Research programs that provide research-based experiences for undergraduate students in science, technology, engineering, and mathematics (STEM) areas have demonstrated a number of benefits, including increases in students' research skills, ability to collaborate when conducting research, and ability to communicate researchbased findings (Bielefeldt 2012; Guerrero, Labrador, and Pérez 2007; Hsieh 2013; Kardash 2000; Lopatto 2007). Undergraduate research programs typically reflect either NSF-funded research experiences for undergraduates (REU) models or comparable REU models sponsored by the university. Despite programmatic differences between the research programs, the overarching goals, intent, and structure of the programs are often similar (Sheng, Landers, and Nguyen 2014; Sutterer et al. 2005; Zydney et al. 2002a). A direct comparison of student research-based experiences and gains based on the type of REU program implemented, however, has not yet been conducted. The primary goal of this research was to examine differences in gains in research-based experiences made by students who participated in an NSF-funded program compared with a university-sponsored REU program.

In a review of the literature, Seymour and colleagues reviewed published studies and conference proceedings examining the impact of undergraduate research experiences on student outcomes (Seymour et al. 2004). Based on their assessment, they clustered the most commonly indicated benefits to students of such programs. These included increased interest in specific areas of research; increased recruitment of underrepresented groups in research-based experiences; increased understanding of the research process; gains in research and researchbased skills; and clarification, refinement, and confirmation of educational- and career-related goals (Seymour et al. 2004). In an evaluation of undergraduate research experiences, Zydney and colleagues found that students 